\title{
Dust accretion onto high-mass planets
}

\author{
S.-J. Paardekooper ${ }^{\star}$ \\ Leiden Observatory, Leiden University, Postbus 9513, 2300 RA Leiden, The Netherlands \\ e-mail: S.Paardekooper@damtp.cam.ac.uk
}

Received 31 August 2006 / Accepted 23 October 2006

\begin{abstract}
Aims. We study the accretion of dust particles of various sizes onto embedded massive gas giant planets, where we take into account the structure of the gas disk due to the presence of the planet. The accretion rate of solids is important for the structure of giant planets: it determines the growth rate of the solid core that may be present as well as their final enrichment in solids.

Methods. We use the RODEO hydrodynamics solver to solve the flow equations for the gas, together with a particle approach for the dust. The solver for the particles' equations of motion is implicit with respect to the drag force, which allows us to treat the whole dust size spectrum.

Results. We find that dust accretion is limited to the smallest particle sizes. The largest particles get trapped in outer mean-motion resonances with the planet, while particles of intermediate size are pushed away from the orbit of the planet by the density structure in the gas disk. Only particles smaller than approximately $s_{\max }=10 \mu \mathrm{m}$ may accrete on a planet with the mass of Jupiter. For a ten times less massive planet $s_{\max }=100 \mu \mathrm{m}$. The strongly reduced accretion of dust makes it very hard to enrich a newly formed giant planet in solids.
\end{abstract}

Key words. hydrodynamics - methods: numerical - planets and satellites: formation

\section{Introduction}

Planets form in circumstellar disks consisting of gas and dust. Initially, gas and solids are well-mixed with a dust-to-gas ratio of $1: 100$, which is similar to the interstellar value, and the dust particles will be as small as interstellar grains $(\sim 1 \mu \mathrm{m})$. However, due to the pressure structure of the disk the dust particles start to move with respect to the gas: the vertical component of the stellar gravity makes the particles rain down onto the midplane of the disk, while the radial pressure gradient of the gas pushes the particles inward (Weidenschilling 1977).

The magnitude of the velocity difference between gas and dust depends on the particle size: small particles couple well to the gas and move slower than the larger particles. These sizedependent velocities in the radial and vertical direction, together with Brownian motion and turbulence, will lead to collisions between the dust particles of various sizes. Assuming that impact velocities are low the particles stick together upon colliding, after which they continue as one large particle. This leads to a rapid depletion of small grains (Dullemond \& Dominik 2005).

However, sticking probabilities are still unclear, especially for larger particles. Experimental results (Blum \& Muench 1993; Blum \& Wurm 2000) suggest that unless the relative velocities of particles are very low collisions lead to bouncing or even shattering of the particles. Another problem arises once the particles grow to sizes of approximately one meter, because then the time scale for drag-induced radial migration becomes as short as 100 yrs for particles at 1 AU (Weidenschilling 1977). These problems led to a renewed interest in planetesimal formation through gravitational instability (Goldreich \& Ward 1973; Youdin \& Shu 2002; Garaud et al. 2004).

* Present address: Department of Applied Mathematics and Theoretical Physics, Wilberforce Road, Cambridge CB3 0WA, UK.
Once the planetesimals reach sizes of roughly $1 \mathrm{~km}$ the aforementioned problems cease to exist. These bodies couple not well enough to the gas to experience fast radial migration, and their gravitational influence is aiding their growth. An important consequence of gravity-aided growth is that the heavier bodies will grow fastest, which leads to a phase of oligarchic growth where a few large bodies dominate the dynamics of the system (Kokubo \& Ida 1998, 2000, 2002). This is an important epoch in planet formation, because it covers the phase from planetesimals to planets of masses comparable to Mercury or Mars in the inner regions of the solar system, while in the outer regions the fullgrown cores of giant planets may have been formed (Chambers 2006). In the core accretion model (Pollack et al. 1996) of giant planet formation these cores of several $M_{\oplus}$ attract significant amounts of gas from the nebula which results in the end in the birth of a gas giant planet.

The alternative for the core accretion model is the gravitational instability scenario, in which a massive disk becomes gravitational unstable and fragments into giant planets (Boss 1997; Mayer et al. 2004). However, whether a disk can become unstable depends sensitively on the cooling time scale (Pickett et al. 2000, 2003) and it is not yet clear if realistic protoplanetary disks will be subject to gravitational instabilities. Furthermore, the fragments produced in numerical simulations have masses around 10 Jupiter masses $\left(M_{\mathrm{J}}\right)$, which is rather high compared to the giant planets found in extrasolar planet surveys.

One of the key differences between the two scenarios is the presence of a solid core. Unfortunately, the existence of solid cores in gas giant planets is still subject of debate. Models of the internal structure of Jupiter (Guillot et al. 2004) can not constrain the core mass from below due to uncertainties in the equation of state near the center of the planet. However, the same models do suggest that Jupiter is enriched in solids with respect to the 
Sun. If Jupiter was formed by means of gravitational instability it would have had to accrete these solids in a later stage.

Accretion of solids also determines the luminosity of forming planets, which is important for future observations of protoplanetary disks. On their way to the core the accreted planetesimals deposit their energy in the gaseous envelope, which radiates away part of this energy into space. This accretion rate plays a major role in the dynamics of forming giant planets (Pollack et al. 1996).

It is therefore important to understand the process of accretion of solids onto forming planets. Greenberg et al. (1978) studied accretion onto solid cores, and the effect of gas drag was investigated by Weidenschilling \& Davis (1985), who found that inward moving planetesimals will be captured in Mean Motion Resonances (MMRs). Only if the gas drag is strong enough (i.e. the planetesimals are small enough) they will make it to the surface of the planet. At first sight one might think that this slows down planetesimal accretion severely, but since their eccentricity is pumped up in the resonances collisions become much more frequent and the small debris resulting of catastrophic collisions subsequently accrete onto the planet (Weidenschilling \& Davis 1985). However, Kary et al. (1993) showed that even particles small enough to move through all resonances do not always reach the surface of the planet. Instead, they are transferred into inferior orbits and they continue to move towards the central star.

These studies including gas drag assumed a uniform gas disk, but hydrodynamical simulations of planets embedded in a gaseous disk show that planets more massive than $0.1 M_{\mathrm{J}}$ start to restructure their environment (e.g. D'Angelo et al. 2002, 2003). Eventually a deep gap forms in the disk for the most massive planets $M_{\mathrm{p}} \geq M_{\mathrm{J}}$ (Lin \& Papaloizou 1993; Bryden et al. 1999). The pressure gradients associated with these gaps dramatically change the behavior of dust particles, which may lead to a dust gap while there is no gas gap (Paardekooper \& Mellema 2004), and also to a substantially reduced accretion rate of solids (Paardekooper \& Mellema 2006a). However, the latter study was limited in particle size and planet mass due to the fluid nature of the simulated dust component. In this paper we use a particlebased method to study dust accretion onto high-mass planets for arbitrary particle sizes. We focus on planets with masses large enough to affect their direct environment, $M_{\mathrm{p}} \geq 0.1 M_{\mathrm{J}}$. This also allows us to do two-dimensional simulations, since the measured gas accretion rates for these planets in $2 \mathrm{D}$ are similar to the 3D values (D'Angelo et al. 2003).

We start in Sect. 2 by reviewing the governing equations of motion, including the adopted gas drag law. In Sect. 3 we describe the numerical method used to integrate the equations of motion, and Sect. 4 is devoted to the disk model. In Sect. 5 we describe the results of the simulations, which we briefly discuss in Sect. 6. We conclude in Sect. 7.

\section{Equations of motion}

Throughout we will work in a cylindrical coordinate frame $(r, \phi)$ with the central star in the origin. Because of the presence of a planet this is not an inertial frame, of which good use has been made in finding extrasolar planets, but we have to correct for this in the potential. The planet's orbit is circular, and the coordinate frame corotates with the planet at angular velocity $\Omega_{\mathrm{p}}$. Our unit of distance is the orbital radius of the planet, which is then located at $(r, \phi)=(1, \pi)$ throughout the simulation. The mass of the planet only enters the problem as a fraction of the stellar mass $q=M_{\mathrm{p}} / M_{*}$. When quoting explicit planetary masses (relative to the mass of Jupiter, $M_{\mathrm{J}}$ ) we assume that the central star has a mass of $1 M_{\odot}$. Then $1 M_{\mathrm{J}}$ corresponds to $q=10^{-3}$.

\subsection{Gas}

The evolution of the gas component of the disk is governed by the Euler equations, which for this specific case are described in detail in Paardekooper \& Mellema (2006b). We do not solve the energy equation, but we use a locally isothermal equation of state:

$p=c_{\mathrm{s}}^{2} \Sigma_{\mathrm{g}}$

where $\Sigma_{\mathrm{g}}$ is the surface density of the gas, $p$ is the vertically integrated pressure, and the isothermal sound speed $c_{\mathrm{s}}$ is directly related to the disk thickness $H$ :

$c_{\mathrm{S}}=H \Omega_{\mathrm{K}}=h v_{\mathrm{K}}$

where $\Omega_{\mathrm{K}}$ is the Keplerian angular velocity, $h=H / r$ and $v_{\mathrm{K}}=$ $r \Omega_{\mathrm{K}}$. The gas has a kinematic viscosity $v$, which we take to be constant throughout the computational domain. We neglect the self-gravity of the gas.

\subsection{Dust particles}

The equations of motion for a dust particle read:

$\frac{\mathrm{d} v}{\mathrm{~d} t}=\frac{L^{2}}{r^{3}}-\frac{\partial \Phi}{\partial r}+f_{r}$

$\frac{\mathrm{d} L}{\mathrm{~d} t}=-\frac{\partial \Phi}{\partial \phi}+f_{\phi}$

where $v$ is denotes radial velocity, $L$ is the specific angular momentum, $\Phi$ is the gravitational potential and $f$ is the drag force, which we specify below. The potential contains contributions from the central star, the planet and indirect terms due to the acceleration of the coordinate frame. We do not consider selfgravity for the dust particles.

\subsection{Drag force}

The nature of the friction between gas and dust depends on the size of the dust particles relative to the mean free path of the gas molecules. This is expressed by the Knudsen number:

$K n=\frac{\lambda}{2 s}$

where $\lambda$ is the mean free path of the gas molecules and $s$ is the size of the dust particles under consideration. When $K n \gg 1$ we are in the Epstein regime of free molecular flow, and the drag force is given by (Schaaf 1963):

$$
\begin{aligned}
\boldsymbol{F}_{\mathrm{eps}}=-\pi s^{2} \rho_{\mathrm{g}}|\boldsymbol{\Delta} v| \boldsymbol{\Delta} v & \\
\times & {\left[\left(1+\frac{1}{m^{2}}-\frac{1}{4 m^{4}}\right) \operatorname{erf}(m)+\left(\frac{1}{m}+\frac{1}{2 m^{3}}\right) \frac{\mathrm{e}^{-m^{2}}}{\sqrt{\pi}}\right] }
\end{aligned}
$$

where $\rho_{\mathrm{g}}$ is the gas density, $\Delta v$ is the velocity of the dust particle relative to the gas, and $m$ is the relative Mach number of the flow: $m=|\Delta v| / c_{\mathrm{s}}$. Equation (6) has the following asymptotic behavior:

$$
\boldsymbol{F}_{\text {eps }}= \begin{cases}-\frac{\sqrt{128 \pi}}{3} s^{2} \rho_{\mathrm{g}} c_{\mathrm{s}} \Delta v & \text { if }|\boldsymbol{\Delta} v| \ll c_{\mathrm{S}} \\ -\pi s^{2} \rho_{\mathrm{g}}|\boldsymbol{\Delta} v| \boldsymbol{\Delta} v & \text { if }|\boldsymbol{\Delta} v| \gg c_{\mathrm{S}}\end{cases}
$$


Because Eq. (6) is difficult to implement numerically we interpolate between these limits in order to obtain the drag force for arbitrary velocities (Kwok 1975):

$\boldsymbol{F}_{\mathrm{eps}}=-\frac{\sqrt{128 \pi}}{3} s^{2} \rho_{\mathrm{g}} c_{\mathrm{s}} f_{\mathrm{D}} \Delta v$

where

$f_{\mathrm{D}}=\sqrt{1+\frac{9 \pi}{128} m^{2}}$.

When $K n \ll 1$ the drag force is given by Stokes friction:

$\boldsymbol{F}_{\text {sto }}=-6 \pi s k_{\mathrm{D}} \mu_{\text {kin }} \Delta v$

where $\mu_{\text {kin }}$ is the kinematic viscosity of the gas, which we write as:

$\mu_{\text {kin }}=\frac{1}{3} \rho_{\mathrm{g}} v_{\mathrm{th}} \lambda$

where $v_{\text {th }}=\sqrt{8 / \pi} c_{\mathrm{s}}$ is the mean thermal velocity of the gas. Note that $\mu_{\text {kin }}$, and therefore also $\boldsymbol{F}_{\text {sto }}$, is independent of the ambient gas density. This in contrast with $\boldsymbol{F}_{\text {eps }}$, for which a higher gas density leads to a larger force of friction.

In Eq. (10) the drag coefficient $k_{\mathrm{D}}$ depends on the Reynolds number $R e$ as follows:

$k_{\mathrm{D}}= \begin{cases}1+0.15 R e^{0.687} & \text { if } \quad R e \leq 500 . \\ 3.9610^{-6} R e^{2.4} & \text { if } 500<R e \leq 1500 . \\ 0.11 R e & \text { if } \quad R e>1500\end{cases}$

where

$R e=\frac{2 s \rho_{\mathrm{g}}|\Delta v|}{\mu_{\mathrm{kin}}}=3 \sqrt{\frac{\pi}{8}} \frac{m}{K n}$.

For flows of intermediate Knudsen number reliable expressions for the drag force are not readily available, so we just interpolate (see Woitke \& Helling 2003):

$\boldsymbol{F}=\left(\frac{3 K n}{3 K n+1}\right)^{2} \boldsymbol{F}_{\mathrm{eps}}+\left(\frac{1}{3 K n+1}\right)^{2} \boldsymbol{F}_{\text {sto }}$.

Using Eqs. (8), (10) and (11) we can rewrite the total drag force as:

$\boldsymbol{F}=-\sqrt{128 \pi} \frac{3 K n f_{\mathrm{D}}+k_{\mathrm{D}}}{(3 K n+1)^{2}} s^{2} \rho_{\mathrm{g}} c_{\mathrm{s}} K n \Delta v$

Upon dividing by the mass of the dust particle, $\frac{4}{3} \pi s^{3} \rho_{\mathrm{p}}$, where $\rho_{\mathrm{p}}$ is the internal density of a dust particle, we can write for the force per unit mass:

$f=-\frac{\Omega_{\mathrm{K}}}{T_{\mathrm{s}}} \Delta v$

where $\Omega_{\mathrm{K}}$ is the Keplerian angular velocity and $T_{\mathrm{s}}$ is the dimensionless stopping time:

$T_{\mathrm{s}}=\sqrt{\frac{\pi}{8}} \frac{(3 K n+1)^{2}}{9 K n^{2} f_{\mathrm{D}}+3 K n k_{\mathrm{D}}} \frac{s}{r} \frac{\rho_{\mathrm{p}}}{\rho_{\mathrm{g}}} \frac{v_{\mathrm{K}}}{c_{\mathrm{s}}}$.

Note that in the limit $|\Delta v| \ll c_{\mathrm{s}}, R e \ll 1$ Eq. (16) is linear in the relative velocity of the particle, which has great advantages for numerically solving the equations of motion (see Sect. 3).

\section{Numerical method}

\subsection{Gas}

We solve the flow equations for the gas using the RODEO method (Paardekooper \& Mellema 2006b). This method was extensively tested on the planet-disk problem, and also used in twofluid mode in Paardekooper \& Mellema (2004). It makes use of an approximate Riemann solver, together with stationary extrapolation (Eulderink \& Mellema 1995) to integrate the source terms. See Paardekooper \& Mellema (2006b) for details.

\subsection{Dust}

The dust component of astrophysical fluids can in principal be described as being a continuous fluid or as a collection of particles. Both approaches have their advantages and disadvantages. The major advantage for the fluid approach is that the flow is accurately described even in regions of very low dust density. In the particle approach these regions would be severely depleted of particles, and therefore the local resolution would be relatively poor. In the context of embedded planets, the opening of a dust gap in a gas disk (Paardekooper \& Mellema 2004) should preferably be modeled using a dust fluid.

However, the fluid approach is not valid in all circumstances (Garaud et al. 2004). First of all, one fluid element (one grid cell) should contain enough dust particles to define a density, and to work with averaged velocities. Second, there should be enough interparticle collisions to make such an average meaningful. In a gas-dominated disk this means that the particles should couple reasonably well to the gas. In view of both these limitations of the fluid approach the larger particles of the dust size distribution can not accurately be modeled as a fluid. The critical size depends on the gas density and the shape of the particles, but for dust grains larger than approximately $10 \mathrm{~cm}$ the fluid approach breaks down at $5 \mathrm{AU}$ in the Minimum Mass Solar Nebula. If locally the gas density is lowered severely, for example by a gapopening planet, this maximum size goes down correspondingly.

Therefore in cases of low gas density the particle approach seems to be the better option, although one has to make sure that enough particles reside inside the gap in order to obtain accurate results. In this study we focus on high-mass planets that open up gas gaps in the disk, and therefore we chose for the particle approach.

In order to integrate the equations of motion numerically we used a standard second order symplectic integrator (leapfrog), in which alternatingly positions and velocities are updated. During a position update, velocities are assumed to be constant and vice versa:

$\frac{\mathrm{d} \boldsymbol{r}}{\mathrm{d} t}=\boldsymbol{v}, \quad \boldsymbol{v}$ constant
$\frac{\mathrm{d} \boldsymbol{v}}{\mathrm{d} t}=\boldsymbol{a}(\boldsymbol{r}, \boldsymbol{v}), \quad \boldsymbol{r}$ constant

where $\boldsymbol{a}$ denotes the acceleration of the particle.

The position update is trivial. For the radial velocity update we need to solve

$\frac{\mathrm{d} v}{\mathrm{~d} t}=\frac{L^{2}}{r^{3}}-\frac{\partial \Phi}{\partial r}-\frac{\Omega_{\mathrm{K}}}{T_{\mathrm{s}}} \Delta v$

Because we keep the gas velocity constant, the same differential equation applies to $\Delta v$. In order to make the integration scheme suited in the regime $T_{\mathrm{s}} \ll 1$ we treat the drag term implicitly. Formally this can only be done in the limit $f_{\mathrm{D}}, k_{\mathrm{D}}=1$ (subsonic 


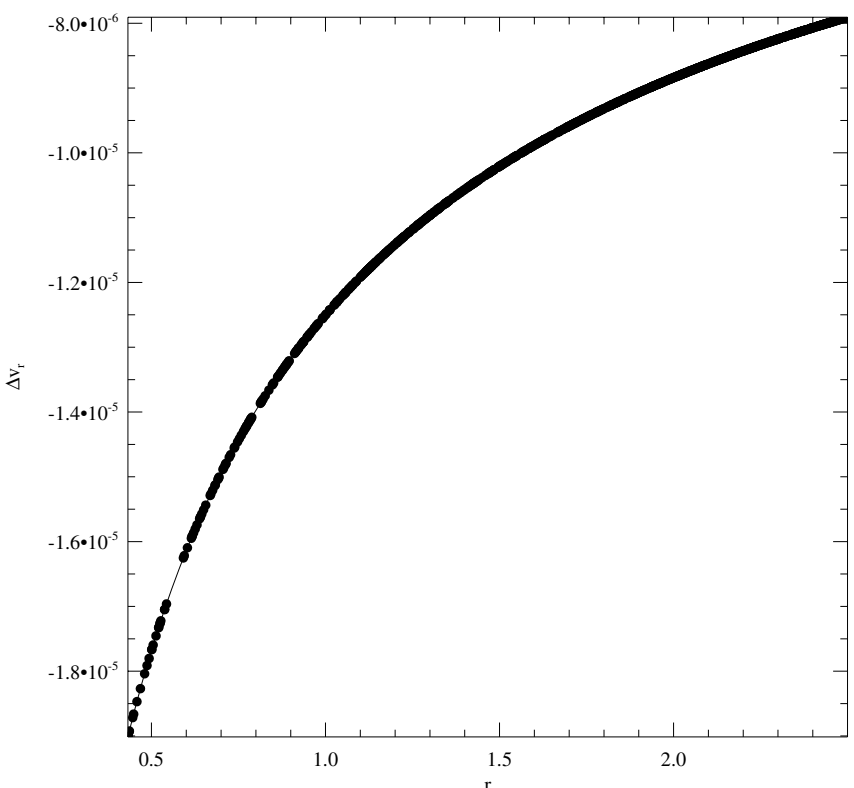

Fig. 1. Radial velocity difference between gas and dust particles with $T_{\mathrm{s}}=0.005$.

and laminar regime). However, because supersonic drift velocities as well as high Reynolds numbers only occur when $T_{\mathrm{s}} \gg 1$ this is not a problem, because then the effects of gas drag are small and there is no need to treat the drag force implicitly.

Upon differencing Eq. (20) we obtain:

$\frac{\Delta v-\Delta v_{0}}{\delta t}=\frac{L^{2}}{r^{3}}-\frac{\partial \Phi}{\partial r}-\frac{\Omega_{\mathrm{K}}}{T_{\mathrm{s}}} \Delta v$

which has the solution

$\Delta v=\frac{\Delta v_{0}+\delta t\left(\frac{L^{2}}{r^{3}}-\frac{\partial \Phi}{\partial r}\right)}{1+\frac{\Omega_{\mathrm{K}} \delta t}{T_{\mathrm{s}}}}$.

A similar equation can be derived for the angular momentum equation. These equations are used to update the radial and angular velocities of the dust particles.

The method was tested in two limiting cases: $T_{\mathrm{S}}=\infty$ and $T_{\mathrm{s}} \ll 1$. When there is no coupling to the gas $\left(T_{\mathrm{s}}=\infty\right)$ the problem reduces to the restricted three-body problem, which has the Jacobi constant as an integral of motion:

$J=E-\mathbf{\Omega}_{\mathrm{p}} \cdot \boldsymbol{L}$

where $E$ is the total energy of the particle under consideration. We reduced the magnitude of the time step for the particle integration with respect to the hydrodynamical time step until $J$ was conserved to 1 part in $10^{6}$ after 100 orbits of the planet. This required 10 particle time steps per hydrodynamical time step. We have adopted this time step for all simulations presented in this paper.

In an axisymmetric disk without a planet, the radial drift velocity is given by (Takeuchi \& Lin 2002):

$\Delta v=-\eta\left(T_{\mathrm{s}}^{-1}+T_{\mathrm{s}}\right) v_{\mathrm{K}}$

where $\eta$ is the ratio of the gas pressure gradient to the stellar gravity. For uniform gas surface density, together with the temperature profile dictated by Eq. (2), $\eta=h^{2}$. We compare this analytical result with the numerical solution for $T_{\mathrm{s}}=0.005$ in Fig. 1, and it turns out that they are indistinguishable. This shows that our implicit integration scheme for the drag force works correctly.

We model dust accretion onto the planet by taking away all particles within one half of the Roche lobe $R_{\mathrm{R}}$ of the planet:

$R_{\mathrm{R}}=\left(\frac{q}{3}\right)^{\frac{1}{3}}$

where $q$ is the ratio of the masses of the planet and the central star. The actual solid core is much smaller than this, so we do not measure the number of impacts onto this core directly. However, the high-mass planets that we consider in this paper are able to capture a dense gaseous envelope that is able to efficiently direct particles towards the center of the planet (see also Paardekooper $\&$ Mellema 2006a). We do not take away gas from the computational domain, as has been done before to mimic gas accretion (Lubow et al. 1999; D'Angelo et al. 2002; Paardekooper \& Mellema 2006b). The process of accretion only influences the density structure close to the planet, and therefore this does not influence the results on dust accretion.

\section{Initial and boundary conditions}

The gas disk model is the same as in Paardekooper \& Mellema (2004). The surface density of gas and dust particles is uniform initially, and we take the gas disk thickness to be $H=0.05 r$, so $h=0.05$, which is the canonical value for simulations of planetdisk interaction. In order to compensate for the radial pressure gradient dictated by Eq. (1) the gas orbits at a slightly subKeplerian angular velocity, while the dust particles are on exact Keplerian orbits initially. We take all initial radial velocities of gas and dust to be zero. The gas has an anomalous viscosity $v$ that is parametrized by the usual $\alpha$-prescription (Shakura \& Sunyaev 1973):

$v=\alpha c_{\mathrm{s}} H$.

We adopt a constant value of $v=10^{-5}$, which corresponds to $\alpha=0.004$ at the location of the planet.

In order to calculate the stopping time in Eq. (17) we need a value for the gas density. We fix the initial density to be $10^{-11} \mathrm{~g} \mathrm{~cm}^{-3}$ at $r=1$, which is appropriate for the location of Jupiter in the Minimum Mass Solar Nebula. From the gas density we can calculate the mean free path of gas molecules:

$\lambda=\frac{m_{\mathrm{H}_{2}}}{\pi \rho_{\mathrm{g}} r_{\mathrm{H}_{2}}{ }^{2}}$

where $m_{\mathrm{H}_{2}}$ and $r_{\mathrm{H}_{2}}$ are the mass and radius of an $\mathrm{H}_{2}$ molecule, respectively. The internal particle density $\rho_{\mathrm{p}}$ is $1.25 \mathrm{~g} \mathrm{~cm}^{-3}$. Using these parameters, we can calculate $T_{\mathrm{s}}$ as a function of particle size $s$ (but note that $T_{\mathrm{s}}$ also depends on $|\Delta v|$ through the factors $f_{\mathrm{D}}$ and $k_{\mathrm{D}}$ ).

The dust density does not enter the equations of motion as long as it remains much smaller than the gas density. If not, the gas is affected by the drag force from the dust particles. We assume that initially the dust-to-gas ratio is equal to $1: 100$, and that we therefore can ignore the feedback on the gas. We vary the number of dust particles $N$ typically from 5000 to $10^{6}$.

The computational domain extends from $r=0.4$ to $r=2.5$ in the radial direction, and from $\phi=0$ to $\phi=2 \pi$ in azimuth. This domain is covered by a uniform grid consisting of 128 radial and 384 azimuthal cells which is used to evolve the gas component of the disk.

We take the boundary conditions for the gas to be nonreflecting (Godon 1996; Paardekooper \& Mellema 2006b), in 



Fig. 2. Density structure after 50 orbits of a $1 M_{\mathrm{J}}$ planet around a solar mass star. Left panel: particle distribution $\left(T_{\mathrm{s}}=0\right), N=5000$. Middle panel: particle distribution $\left(T_{\mathrm{s}}=0\right), N=10000$. Right panel: logarithm of the gas surface density.

order to keep the waves generated by the planet from reflecting back into the computational domain. When a dust particle moves off the computational domain we remove it from the simulation.

\section{Results}

We have performed simulations with different particle sizes in order to sample the parameter space in stopping time ranging from the limit of perfect coupling $T_{\mathrm{s}}=0$ to the limit of no coupling $T_{\mathrm{s}}=\infty$. The former limit offers an opportunity to compare the measured accretion rates to the gas accretion, so we will start by discussing perfectly coupled particles.

When we increase the size of the particles the stopping time increases as well. We can distinguish three regimes:

- $T_{\mathrm{s}} \ll 1$ : the motion of the particles is dominated by gas drag. However, significant gas-dust separation may occur in the presence of planets on time scales of approximately $\Omega_{\mathrm{K}}^{-1} T_{\mathrm{s}}^{-1}$ (see Paardekooper \& Mellema 2004).

- $T_{\mathrm{s}} \gg 1$ : particle motion is dominated by gravitational interaction with the star and the planet, with the gas drag as a small perturbation.

- $T_{\mathrm{s}} \approx 1$ : the orbital time scale for a particle is comparable to the time scale for gas drag. Depending on the gas density, there may be region in which either gas drag or gravity will dominate.

\subsection{Perfect coupling}

First of all we discuss the case for $T_{\mathrm{s}} \ll \Omega_{\mathrm{K}} \Delta t$, where $\Delta t$ is the magnitude of the time step as required for the integration of the Euler equations. For such a small value of the stopping time the dust particles are forced to move with the gas velocity, and the dust-to-gas ratio remains the same everywhere. In particular, gas and dust accretion rates should be equal as well, when we correct for the factor 100 in the dust-to-gas ratio.

Gas accretion onto embedded planets has been studied before using different numerical approaches (Lubow et al. 1999; D'Angelo et al. 2002; Paardekooper \& Mellema 2006b), and they were found to agree reasonably well with each other. There is no strong dependence on the details of the accretion procedure (differences remain within $\sim 40 \%$ ), except for intermediate mass planets for which the Roche lobe is approximately equal to the disk scale height. For these planets, accretion depends strongly on the conditions inside the Roche lobe and the measured accretion rates differ up to a factor of 2 . Therefore we focus on a $1 M_{\mathrm{J}}$ planet, for which a well-defined gas accretion rate has been measured of $\dot{M}=10^{-4}$ disk masses per orbit; a value that we would like to reproduce for the perfectly coupled dust particles. Typically this value was reached within 200 orbits of the planet.

We have varied the number of particles in the simulation from 5000 to $10^{5}$. Note that in order to accurately measure an accretion rate the number of particles that is accreted onto the planet every orbit should be larger than one. For an accretion rate of $10^{-4}$ disk masses per orbit the total number of particles in the simulation $N$ therefore needs to be larger than $10^{4}$. For a smaller value of $N$ we need to rebin the accreted particles into larger time intervals. The loss of time resolution is of no importance for the final accretion rate because it does not vary rapidly after $\sim 50$ orbits.

In Fig. 2 we show the particle distribution for a simulation of a $1 M_{\mathrm{J}}$ planet after 50 orbits. In the gas density (right panel) we see the gap that is developing, as well as the prominent spiral waves excited by the planet. For a run with 10000 particles (middle panel) we can clearly see the gap and also the spiral waves in regions where the particle density is high enough. However, in the gap region near the planet, where in the gas density we see the origin of the spiral waves, nothing is to be seen in the particle density. This is because the spatial resolution in a particle simulation critically depends on the particle density. In regions of low density, as in the case of a disk gap, the resolution is correspondingly low and the spiral waves are not visible. This is of no concern for the outcome of these simulations, however, because the dust density has no dynamical effect. If the density was used for example in calculating the number of collisions between these particles, which in turn would create smaller dust grains, resolution effects would play an important role. The same would be true if the dust density becomes high enough that the gas feels its drag. The only thing we need to worry about for these 


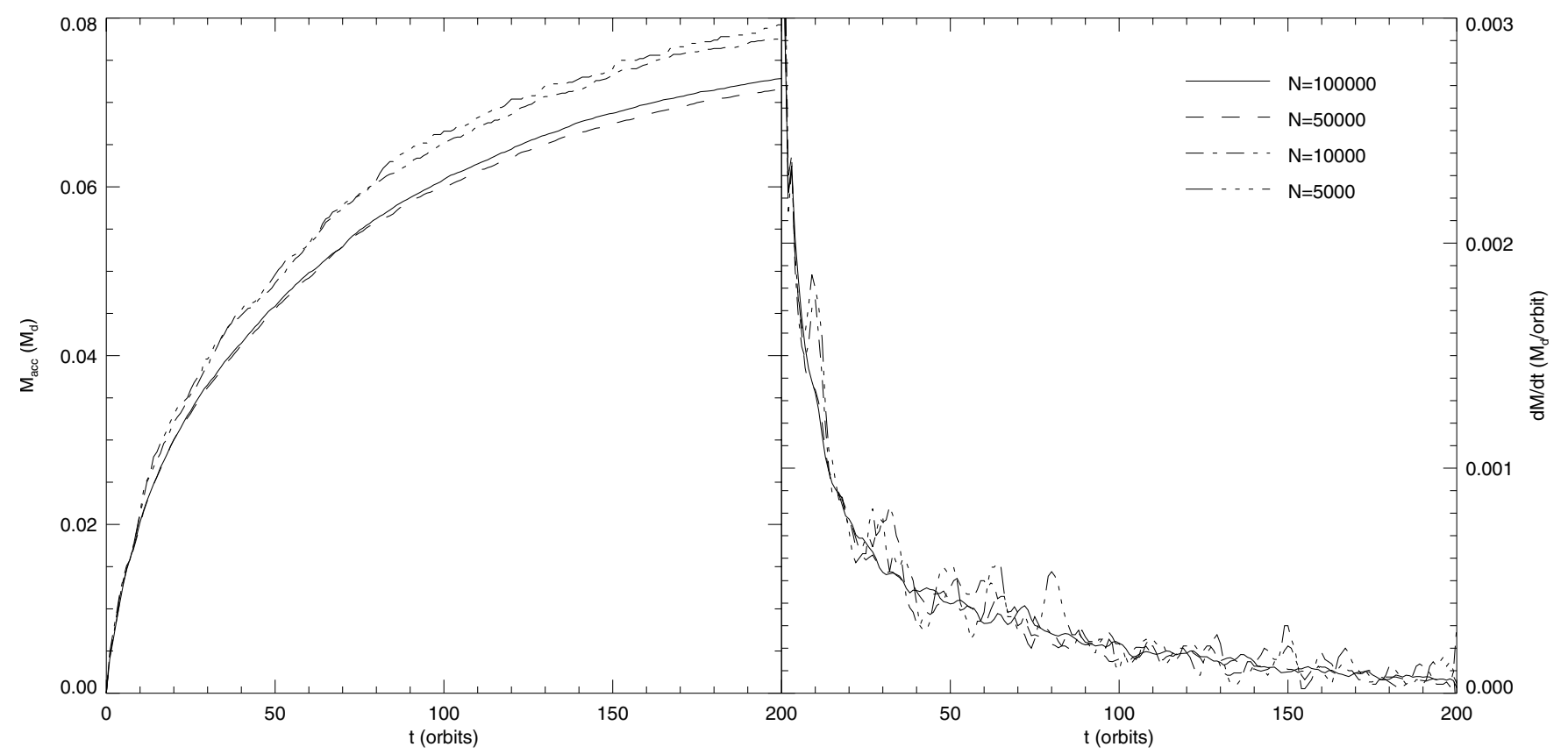

Fig. 3. Accretion of perfectly coupled particles onto a $1 M_{\mathrm{J}}$ planet for four different amounts of particles. Left panel: accreted dust mass in units of the total dust mass in the disk. Right panel: corresponding accretion rates, in disk masses per orbit.

simulations is that we accrete enough particles per time step (see above).

Even if we lower the number of particles $N$ to 5000 there is still density structure visible (left panel of Fig. 2). The outer edge of the gap is less sharp than in the case of $N=10000$, but all features in the density can still be identified.

Also, if we look at the accretion rates for different values of $N$ in Fig. 3 the results look promising, as expected. After 200 orbits, the amount of disk mass that was accreted for the various number of simulated particles differs less than $15 \%$. Even more, the final accretion rates agree very well, yielding a mean value of $\dot{M}=9.5 \times 10^{-5}$ disk masses per orbit, in close agreement with the value that came out of gasdynamical simulations. The total mass that is accreted as well as the slightly lower accretion rates can be attributed to the large imposed accretion inside the Roche lobe. While for the gas case one usually takes out a small fraction of the density away from the accretion region every time step, for our particle simulations we remove all particles from the inner half of the Roche lobe. Such a high imposed accretion rate tends to decrease the total amount of mass that is accreted (see Fig. 7 of Paardekooper \& Mellema 2006b).

We conclude that we are able to reproduce the gas accretion rates in the limit of perfectly coupled particles, and that we only need a relatively small amount of particles to achieve this.

\subsection{No coupling}

In this section we will focus on the limit of no coupling to the gas. For these particles the problem is equivalent to the restricted three-body problem, and therefore we can gain some insight by examining the integral of motion of the problem, $J$ (see Eq. (23)). In particular, the motion of a particle is confined to a region bound by so-called zero-velocity curves, which can be obtained by solving Eq. (23) for a given value of $J=J_{0}$ and all velocities equal to zero. Particles with a starting value $J=J_{0}$ will never cross the zero-velocity curve associated with $J_{0}$. This means that the region in the disk from which the planet may

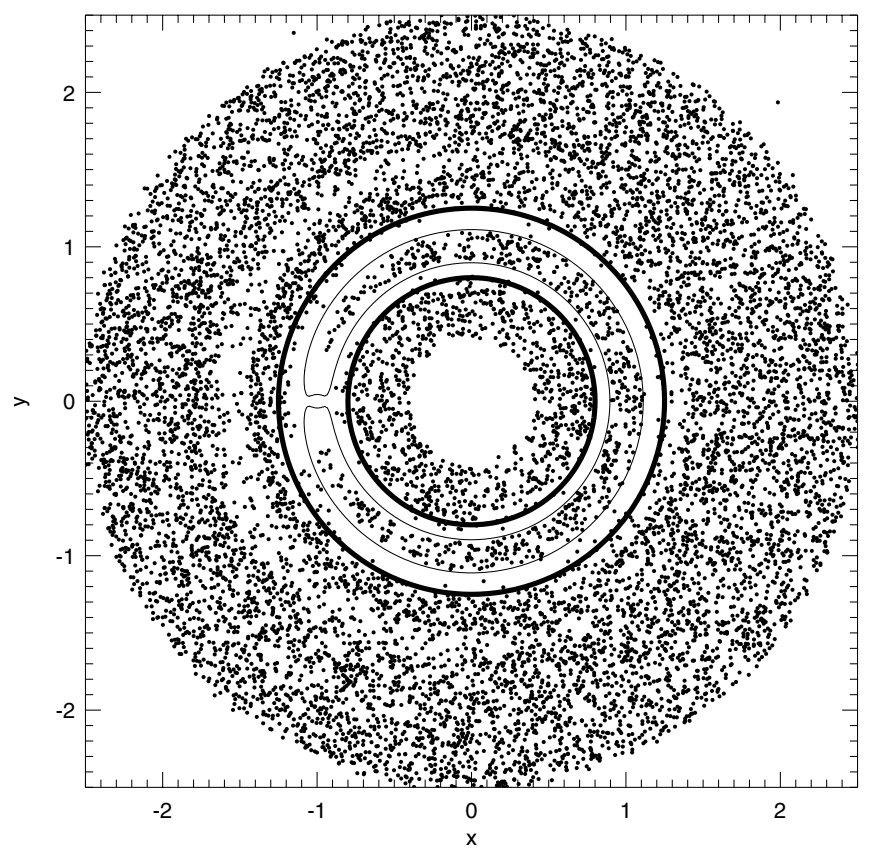

Fig. 4. Particle distribution $(N=10000)$ for $T_{\mathrm{s}}=\infty$ after 500 orbits of a $1 M_{\mathrm{J}}$ planet. Thick contours mark the boundary of the accretion region of the planet. The thin contour gives the corresponding zero-velocity curve.

accrete particles is also bounded, and once this feeding zone is empty accretion will stop.

In Fig. 4 we show the particle distribution after 500 orbits of the planet for $T_{\mathrm{s}}=\infty$ and $N=10000$. The region close to the planet is almost empty, and two annular empty rings appear at approximately $r=1.1$ and $r=0.9$. Particles in these rings travel on horseshoe orbits and suffer close encounters with the planet, which leads to accretion of these particles. Material that orbits further away from the planet $(r>1.1$ and $r<0.9)$ can not reach the planet because of the constraint set by the Jacobi 


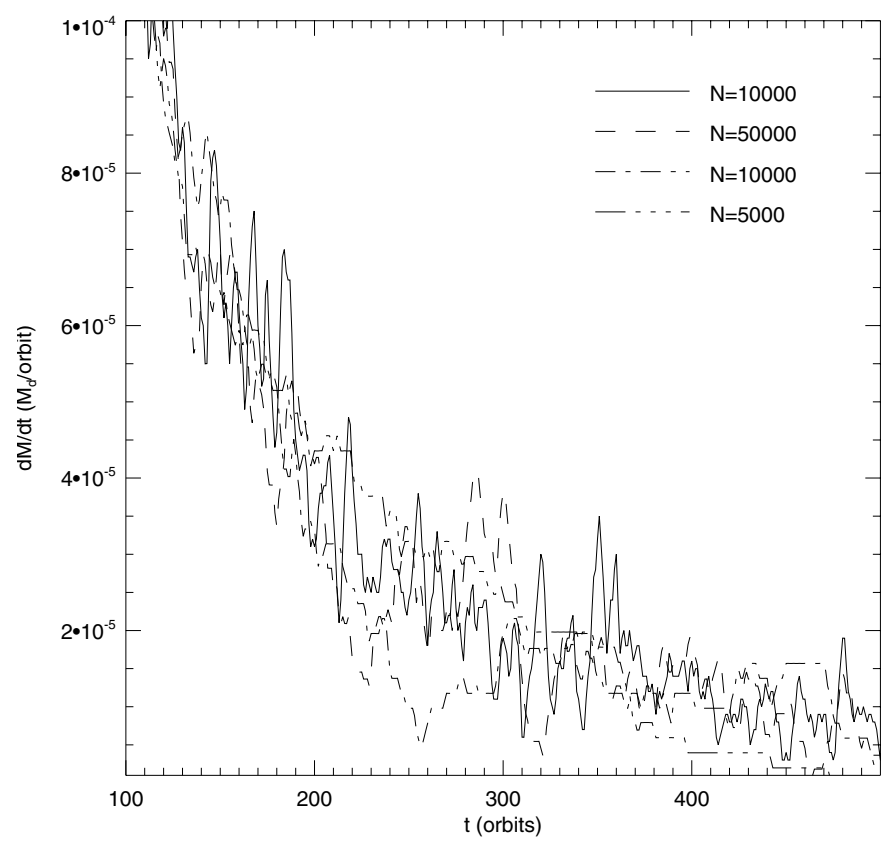

Fig. 5. Accretion of uncoupled particles onto a $1 M_{\mathrm{J}}$ planet for four different amounts of particles.

integral. The thick contours mark the radii at which particles may just make it to the planet, which is shown by the zero-velocity curve (thin contour) corresponding to the Jacobi constant at the location of the thick contour.

The thick contours trace the outer edges of the feeding zone reasonably well, and almost all particles initially located just inside this feeding zone are accreted by the planet. However, particles closer to $r=1$ do not suffer close encounters with the planet: they move on stable tadpole orbits and will not be accreted. In the solar system similar objects can be found around the orbit of Jupiter: the Trojan asteroids. The end result of the simulation is a particle distribution with two empty "rails" at a radial distance of 0.1 from the planet, together with a non-empty corotation region. The empty semi-circle outside the orbit of the planet is due to resonant perturbations of the $2: 1$ outer mean motion resonance.

In Fig. 5 we show the accretion rate up to 500 orbits for the same simulation, for four different values of $N$. Before $t=$ 100 (not shown in the figure) the accretion rate is very high, and the planet accretes roughly $10 \%$ of the total disk during that time. However, after approximately 120 orbits the accretion rate drops below the gas accretion rate $\left(10^{-4}\right.$ disk masses per orbit) and it continues to decline for the whole simulation time. After 500 orbits the particle accretion rate is an order of magnitude below the gas accretion rate, which makes further dust accretion negligible.

All particles were started on circular orbits until now, while mutual gravitational interactions between larger boulders leads to eccentricity excitation (Greenzweig \& Lissauer 1990, 1992). The eccentricity of the particles' orbits determines the width of the accretion zone, and therefore the total mass that may be accreted. However, because the feeding zone is still bounded the accretion rate will still tend to zero after a certain amount of time. This is illustrated in Fig. 6, where we show the accretion rates for three different initial eccentricity distributions. All eccentricities in the specified range were uniformly distributed amongst the particles. It is clear that from approximately 130 orbits the accretion rate is the same for all initial eccentricities, which is the same time scale as for the clearing of the feeding zone (see above). Therefore a non-zero initial eccentricity for the planetesimals enlarges the accretion zone, and therefore increases the total amount of mass that can be accreted (see the left panel of Fig. 6), but it does not affect the time scale on which the feeding zone is cleared.

\subsection{Steady gas disk}

Clearly, the feeding zone needs to be replenished one way or the other in order for accretion to continue. In this section, we explore the possibility that gas drag may prevent the feeding zone from getting empty. We do this by keeping the gas disk steady, in order to separate the effects on accretion of a non-uniform gas disk from effects due to pure dust dynamics. Also this gives us an opportunity to compare with previous work by Kary et al. (1993).

Based on Eq. (24) one might expect that dust accretion would always be stronger than gas accretion. Because the mass that flows inward through a circle with radius $r$ equals:

$\dot{M}=-2 \pi r \Sigma v_{r}$

the ratio of gas and dust accretion rates would be given by:

$\frac{\dot{M}_{\mathrm{d}}}{\dot{M}_{\mathrm{g}}}=\frac{2 \pi r \Sigma_{\mathrm{d}} v_{r, \mathrm{~d}}}{2 \pi r \sum_{\mathrm{g}} v_{r, \mathrm{~g}}}=d\left(1+\frac{\Delta v}{v_{r, \mathrm{~g}}}\right)$

where $d$ is the dust-to-gas ratio and $\Delta v$ is given by Eq. (24). In an unperturbed disk with constant surface density and kinematic viscosity $v$ the radial velocity of the gas is equal to $v_{r, \mathrm{~g}}=-\frac{3 v}{2 r}$, and therefore we have:

$\frac{\Delta v}{v_{r, \mathrm{~g}}}=\frac{h^{2}\left(T_{\mathrm{s}}^{-1}+T_{\mathrm{s}}\right) v_{\mathrm{K}}}{\frac{3}{2} \alpha c_{\mathrm{s}} h}=\frac{2\left(T_{\mathrm{s}}^{-1}+T_{\mathrm{s}}\right)}{3 \alpha}$.

When all dust grains have the same stopping time $T_{\mathrm{s}}$ the gas-dust mixture that is accreted by the planet has an effective dust-to-gas ratio of

$d^{*}=d\left(1+\frac{2\left(T_{\mathrm{s}}^{-1}+T_{\mathrm{s}}\right)}{3 \alpha}\right)$

Using $\alpha=0.004$, already for $T_{\mathrm{s}}=0.001$ the planet would get enriched in solids by more than $15 \%$.

However, the planet may not be able to grab all the material that is passing by (Kary et al. 1993) which leads to lower dust accretion rates. Gas, however, may be accreted at a higher rate than predicted by Eq. (28) (see Lubow et al. 1999; Lubow \& D'Angelo 2006). It is therefore not clear, even in an unperturbed gas disk, how dust accretion compares to gas accretion.

In Fig. 7 we show the dust accretion rates of dust particles onto a $1 M_{\mathrm{J}}$ planet as a function of particle size. Note that we do not take into account effects of the particle size distribution in the disk: the accretion rates shown would be valid if all particles in the disk would have a single size. The solid line gives the accretion rate predicted by Eq. (28) with the radial velocity of Eq. (24). Towards the lowest values of $s$ (and therefore the lowest values of $T_{\mathrm{s}}$ ) it approaches the value of the gas accretion. Note, however, that this is not the measured gas accretion rate, which is higher due to the high accretion efficiency of the planet (Lubow et al. 1999).

Particles $T_{\mathrm{s}} \ll 1$ have a small radial velocity, and they can essentially all be captured by the planet. This results in Fig. 7 in accretion rates close to the predicted value for the smallest 




Fig. 6. Accretion of uncoupled particles $(N=5000)$ onto a $1 M_{\mathrm{J}}$ planet for three different initial eccentricity distributions. Left panel: accreted dust mass in units of the total dust mass in the disk. Right panel: corresponding accretion rates, in disk masses per orbit.



Fig. 7. Accretion of particles onto a $1 M_{\mathrm{J}}$ planet. The solid line gives the accretion rate of dust particles predicted by Eq. (29). All runs included 5000 particles.

particles. When $T_{\mathrm{s}}$ increases towards 1 , the radial velocity of the dust particles increases, but at the same time is is easier for the planet to accrete them because they suffer no strong gas friction. Therefore essentially all particles with $T_{\mathrm{s}}<1$ can be accreted by the planet.

For larger particles a dramatic change in accretion rate occurs. Increasing the size of the particles by less than a factor of three leads to a reduction of accretion by almost two orders of magnitude. The reason for this low accretion rate lies in the mechanism of resonance trapping (Weidenschilling \& Davis 1985), where inward moving particles are captured in mean

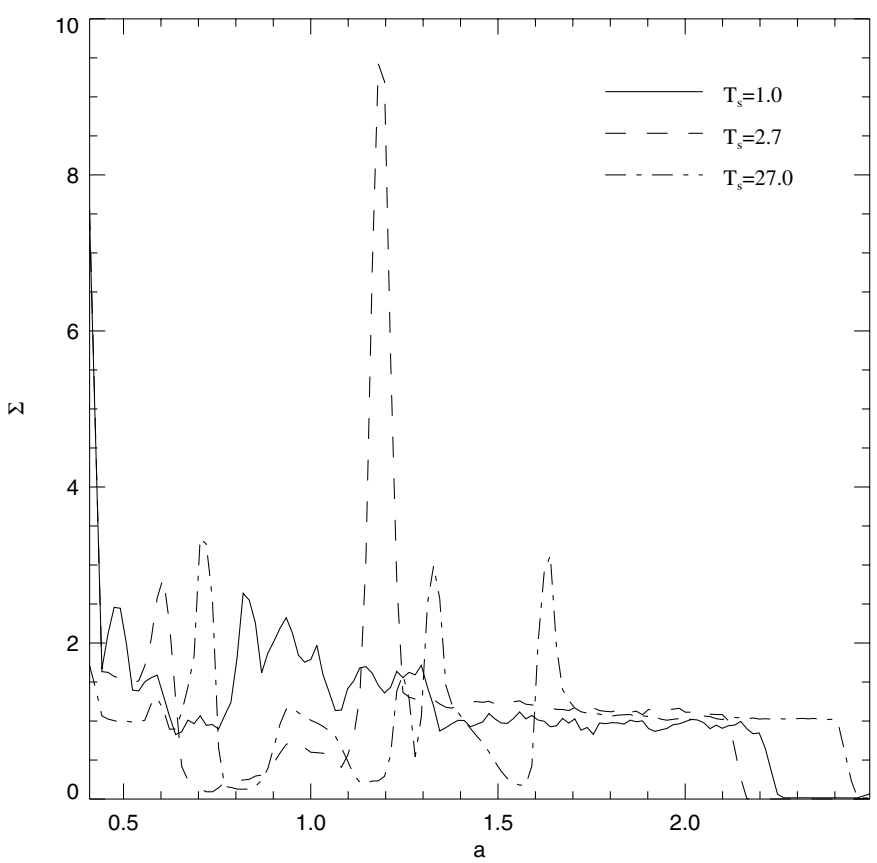

Fig. 8. Particle surface density after 50 orbits of a $1 M_{\mathrm{J}}$ planet and three different values for $T_{\mathrm{s}}$.

motion resonances (MMRs). The drag force that moves the particles inward is exactly balanced by resonant perturbations which are directed away from the planet. This is illustrated in Fig. 8, where we show azimuthally averaged particle surface density as a function of particle semi-major axis $a$. Because the eccentricities remain below 0.1 (see Fig. 9), $a$ almost coincides with $r$.

The solid line in Fig. 8 corresponds to the particles size responsible for the peak of the accretion rate in Fig. 7, $s=37.0$. There is a lot of structure near the position of the planet, but no gap is forming and there is no structure in the outer disk. Note that the surface density goes to zero at $a \approx 2.25$ because of the 


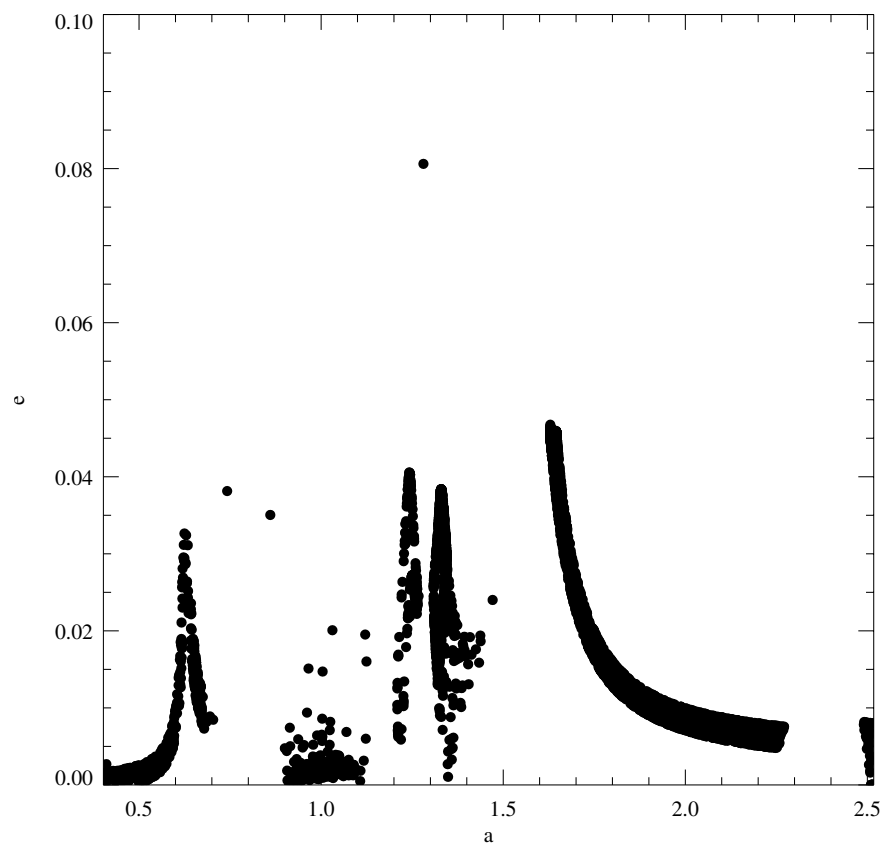

Fig. 9. Particle semi-major axis versus eccentricity after 200 orbits of a $1 M_{\mathrm{J}}$ planet and $s=1000 \mathrm{~cm}\left(T_{\mathrm{s}} \approx 27.0\right)$.

movement of the particles towards the central star. In the inner disk a small feature is visible at $a=0.48$, which we can attribute to the 1:3 MMR. Note, however, that these structures in the inner disk are not stable: resonant perturbations as well as gas drag both push the particles inward.

When we increase the particle size by less than a factor of three (dashed line in Fig. 8) the situation is remarkably different. Near $a=1$ an annular gap starts to form, together with a strong overdensity at $a=1.2$, which is close to the $4: 3$ mean motion resonance $(a \approx 1.21)$. Apart from this large peak there is no structure in the outer disk. The inner disk shows a feature near the $1: 2$ MMR at $a=0.63$.

Increasing the particle size by another factor of 10 again changes the picture (dash-dotted line in Fig. 8). Now the feature at $a=1.2$ has disappeared and instead we can see a peak near the 2:1 MMR at $a=1.6$, together with a feature at $a=1.3$, which corresponds to the 3:2 MMR. In the inner disk the 2:3 MMR is excited at $a=0.76$.

It is clear from Fig. 8 that resonance trapping occurs when $T_{\mathrm{s}}>1$, and that weaker-coupled particles may occupy lowerorder resonances. Close to a resonance the eccentricity of a particle is excited. In Fig. 9 we show the eccentricity $e$ of the particles' orbits for the $T_{\mathrm{s}}=27.0$ case from Fig. 8. At the location of the 2:1 MMR $e$ is excited to a maximum of approximately 0.05 , which is consistent with the analytical result of Weidenschilling \& Davis (1985) who showed that the equilibrium eccentricity for a particle in a $(j+1): j$ MMR is approximately given by $\bar{e}=0.07 / \sqrt{j+1}$.

Resonance trapping occurs because resonant perturbations tend to change the semi-major axis of the particle's orbit in such a way that it moves away from the planet. For outer resonances, this means that this effect opposes the drag-induced inward migration. Inner resonances can never trap particles in a stable way, unless the drag force is directed outward. This may happen when a strong gas density gradient is present, possibly at the outer edge of a gas gap in the disk.

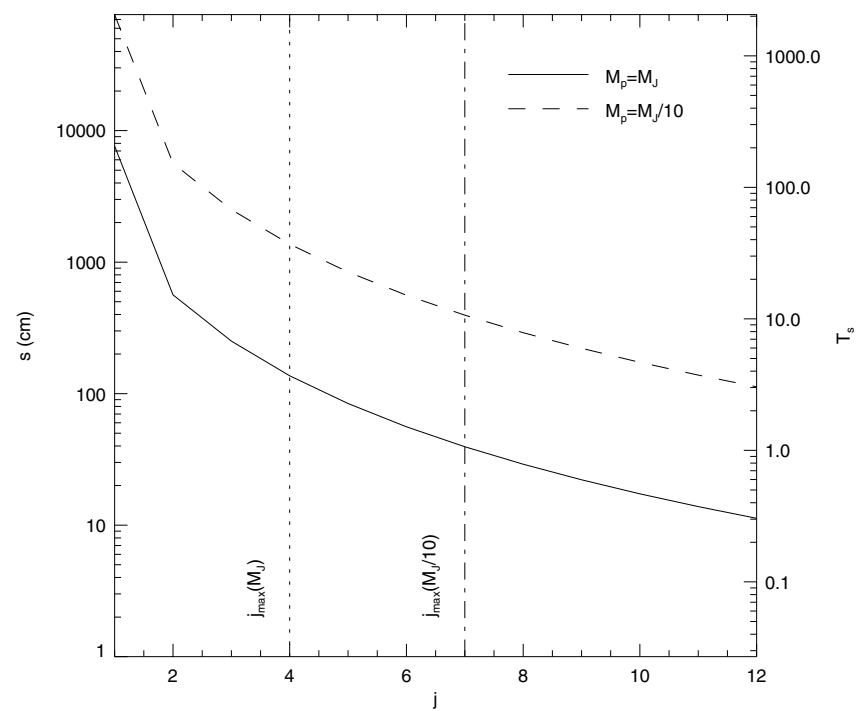

Fig. 10. Minimum particle size that can be trapped in the $j$ th resonance, for a $1 M_{\mathrm{J}}$ planet and a $0.1 M_{\mathrm{J}}$ planet. The vertical lines mark the onset of chaotic orbits for high $j$.

The strength of a resonance $(j+1): j$ depends on the mass planet-to-star mass ratio $q$ as well as on the order of the resonance $j$. Higher order resonances, which are located closer to the planet, are stronger and they can stop inward moving particles more easily. Therefore the location at which a particle is stopped by resonant perturbations depends on the particle size $s$. This is clear from Fig. 8, where particles with $T_{\mathrm{s}}=27.0(\mathrm{~s}=1000 \mathrm{~cm})$ become trapped in the $j=1$ resonance, while particles with $T_{\mathrm{s}}=2.7(\mathrm{~s}=100 \mathrm{~cm})$ move all the way to the $j=3$ resonance. Weidenschilling \& Davis (1985) derived a minimum size for a particle that is able to move through the $j$ th resonance:

$s_{\min }=\frac{\rho_{\mathrm{g}} h r_{\mathrm{p}}}{3 \rho_{\mathrm{p}} q C(j) j^{3 / 2}}$

where $C(j)$ is an increasing function of $j$. For particles smaller than $s_{\min }$ the drag force is stronger than the resonant perturbations and the particles are able to move inward through the resonance. Because $s_{\min }$ becomes smaller for higher values of $j$ they may get trapped in a resonance closer to the planet.

For higher values of $j$, successive resonances are more closely spaced and their overlap may lead to chaotic behavior (Wisdom 1980). The distance at which this happens is approximately (Duncan et al. 1989):

$\left|r-r_{\mathrm{p}}\right| \approx 1.5 q^{2 / 7}$

For a planet of $1 M_{\mathrm{J}}$ this means that particles cannot be trapped closer than $\left|r-r_{\mathrm{p}}\right|=0.2$. It is interesting to note that this is also approximately equal to the width of the gas gap that is opened by a planet of the same mass (see Bryden et al. 1999; Paardekooper \& Mellema 2006b).

In Fig. 10 we show $s_{\min }$ as a function of $j$ for a $1 M_{\mathrm{J}}$ planet and a $0.1 M_{\mathrm{J}}$ planet. Also shown are the maximum values of $j$ for which no chaotic orbits exist (from Eq. (33)). Focusing on the $1 M_{\mathrm{J}}$ planet we see that only particles smaller than approximately $100 \mathrm{~cm}$ are able to travel through the $j=4$ resonance, beyond which no stable trapping exists because of Eq. (33). In terms of accretion onto the planet this means that only particles smaller than $100 \mathrm{~cm}$ are sufficiently coupled to the gas to make it all the way to the surface of the planet. From Fig. 7 we see that indeed the large jump in accretion rate happens around 


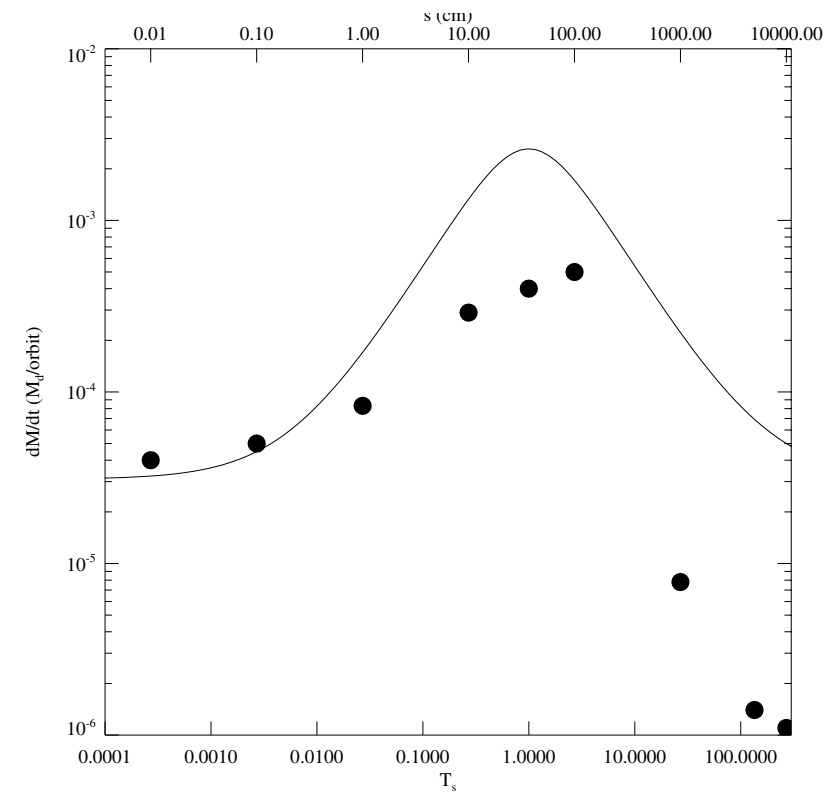

Fig. 11. Accretion of particles onto a $0.1 M_{\mathrm{J}}$ planet.The solid line gives the accretion rate of dust particles predicted by Eq. (29).

$s=100 \mathrm{~cm}$. Our numerical result for $s_{\min }$ agrees within a factor of 2 with Eq. (32).

From Fig. 7 it is clear that essentially all particles that make it through the resonances are eventually accreted by the planet. This is not true in general (Kary et al. 1993), and especially for low-mass planets a significant fraction of particles that cross the orbit of the planet are transferred to the inner disk rather than to be accreted onto the planet. This is illustrated for the case of $q=10^{-4}$ in Fig. 11. Moving from the smallest towards the largest particles (from left to right in Fig. 11) we see three classes of particles:

I. Particles with $s<1 \mathrm{~cm}$ are very strongly coupled to the gas, which makes their radial velocity low enough for them to always reach the planet.

II. Particles with $s>1000 \mathrm{~cm}$ are again trapped in exterior resonances, and the accretion rate is therefore very low.

III. In between, there is a steady flow of particles onto the planet but there is a significant fraction of particles that misses the planet.

The transition from Class III to Class II happens again approximately at the size predicted by Eq. (32) (see also Fig. 10). The deviation from the accretion rate given by Eq. (29) for Class III particles seems to increase with $s$, except for the largest Class III particle at $s=100$, which has a relatively high accretion rate.

From Figs. 7 and 11 we conclude that particles that are trapped in exterior resonances are excluded from accretion onto the planet. Only particles smaller than $s_{\min }$ may reach the planet, where $s_{\min }$ is approximately given by Eq. (32). Particles with for which $1 \mathrm{~cm} \leq s \leq s_{\min }$ potentially have a higher accretion rate than the gas.

\subsection{Evolving gas disk}

However, when planets get massive enough to attract significant amounts of gas and dust they also start to reshape the surrounding disk. Planets as massive as Jupiter open up a deep gas gap in the disk, but already Neptune-class planets restructure the gas disk in such a way that a dust gap emerges, even though there is no gap in the gas (Paardekooper \& Mellema 2004). It was also shown that this leads to a dramatic decline in dust accretion (Paardekooper \& Mellema 2006a) for particles larger than $0.1 \mathrm{~cm}$. In this section we want to study the general case of dust accretion onto high-mass planets.

We start by inspecting the particle distribution after 20 orbits of a $1 M_{\mathrm{J}}$ planet in Fig. 12. From the upper left panel to the lower right panel the stopping time increases from $T_{\mathrm{s}}=0$ (perfectly coupled particles, see Sect. 5.1) to $T_{\mathrm{s}}=\infty$ (uncoupled particles, see Sect. 5.2). After 20 orbits the planet did not get a chance to open up a gap in the gas yet, as is clear from the upper left panel. The uncoupled particles in the lower right panel also do not clear a whole gap, but instead two empty "rails" (see also Fig. 4).

For particles with $T_{\mathrm{s}}=0.027$ (upper-middle panel in Fig. 12) we see that the pressure gradients at the edges of the forming gap are pushing the particles away from the planet's orbit, as was also observed in Paardekooper \& Mellema (2004) for a ten times less massive planet. Because of the high mass of the planet in Fig. 12, and the corresponding large pressure gradients, the effect takes place on much shorter time scales. Note also that because of the forming gap the gas density is already significantly lower near the orbit of the planet, and consequently the particles couple not as well to the gas as at the start of the simulation. This, together with the larger pressure gradients at the gap edges accounts for the fast evolution of the dust component of $1 \mathrm{~cm}$.

When the stopping time approaches 1 the drift velocity of the particles becomes so large that already after 20 orbits of the planet an almost empty gap has formed (upper right and lower left panel of Fig. 12). The particles accumulate at the gap edges and at corotation. The latter because when a gas gap is forming the density at $r=1$ is always larger than at $|r-1|=0.1$ (see Kley 1999; Paardekooper \& Mellema 2006b), and the corresponding pressure gradient pushes the dust particles towards $r=1$. However, when the gas gap is sufficiently clean (which happens after approximately 100 orbits) this pressure gradient disappears and the particles near $r=1$ will be dragged inward.

When $T_{\mathrm{s}}>1$ (lower middle panel in Fig. 12) we see again that the pressure gradient associated with the edges of the gas gap stimulate the formation of the two empty "rails" compared to the case of no coupling. The first signs of resonant capture can be seen as a dark eccentric ring outside the planet's orbit. Because these particles do not couple well to the gas it becomes progressively harder to clean the corotation region while the gas gap is forming. Particles larger than $1000 \mathrm{~cm}$ are not removed from the corotation region before the gas gap is complete, which makes the horseshoe-shaped feature at $r=1$ almost permanent just as in the case of no coupling.

At later times, resonant trapping comes to dominate the density structure of the largest particles. In Fig. 13 we show the azimuthally averaged surface density after 100 orbits of the planet for the gas and for three different particle sizes. The first thing that catches the eye is the large peak in the density of particles with $s=37 \mathrm{~cm}$. The gas density (solid line in Fig. 13) reveals that the pressure gradients around the peak all point away from $r=1.4$. This makes this location an efficient dust trap for basically all material originally located at $1.1<r<1.8$. Locally the surface density is increased with a factor of 25 .

The smaller particles $(1 \mathrm{~cm})$ move slower with respect to the gas than the $37 \mathrm{~cm}$ particles. As a result two peaks can be seen near $r=1.4$ : one from outward moving particles coming from $r \approx 1$ and one from inward moving particles coming from $r>1.7$. The large particles of $1000 \mathrm{~cm}$ also show a doublepeaked feature. Note that the density gradient near the $2: 1 \mathrm{MMR}$ at $r=1.6$ is such that the particles are dragged inward more 




Fig. 12. Particle distribution after 20 orbits of a $1 M_{\mathrm{J}}$ planet for 6 different particle sizes. Top row, from left to right: $s=0$ (perfect coupling, $\left.T_{\mathrm{s}}=0\right), s=1.0 \mathrm{~cm}\left(T_{\mathrm{s}}=0.027\right)$ and $s=10.0 \mathrm{~cm}\left(T_{\mathrm{s}}=0.27\right)$. Bottom row, from left to right: $s=37.0 \mathrm{~cm}\left(T_{\mathrm{s}}=1.0\right), s=1000.0 \mathrm{~cm}\left(T_{\mathrm{s}}=27.0\right)$ and $s=\infty$ (no coupling, $T_{\mathrm{s}}=\infty$ ).

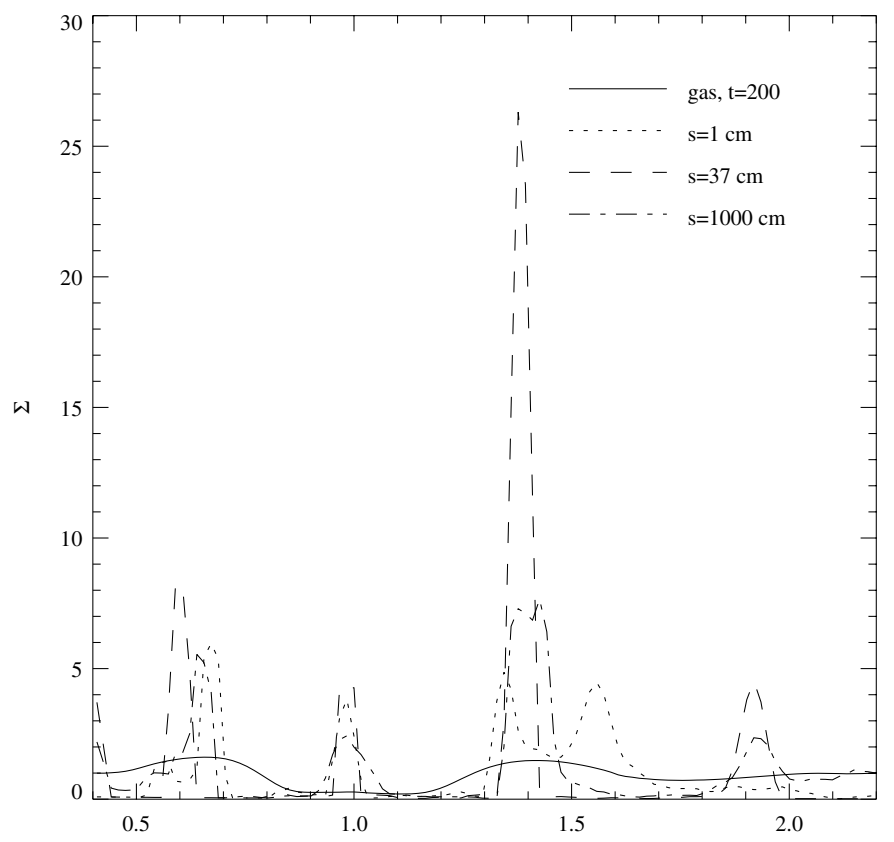

Fig. 13. Azimuthally averaged surface density of gas and dust after 100 orbits of a $1 M_{\mathrm{J}}$ planet for three different particle sizes.

efficiently than in the case of the steady gas disk. Therefore while Fig. 10 predicts that the $1000 \mathrm{~cm}$ particles should be captured in the $2: 1 \mathrm{MMR}$ they are able to move all the way to $r \approx 1.4$.

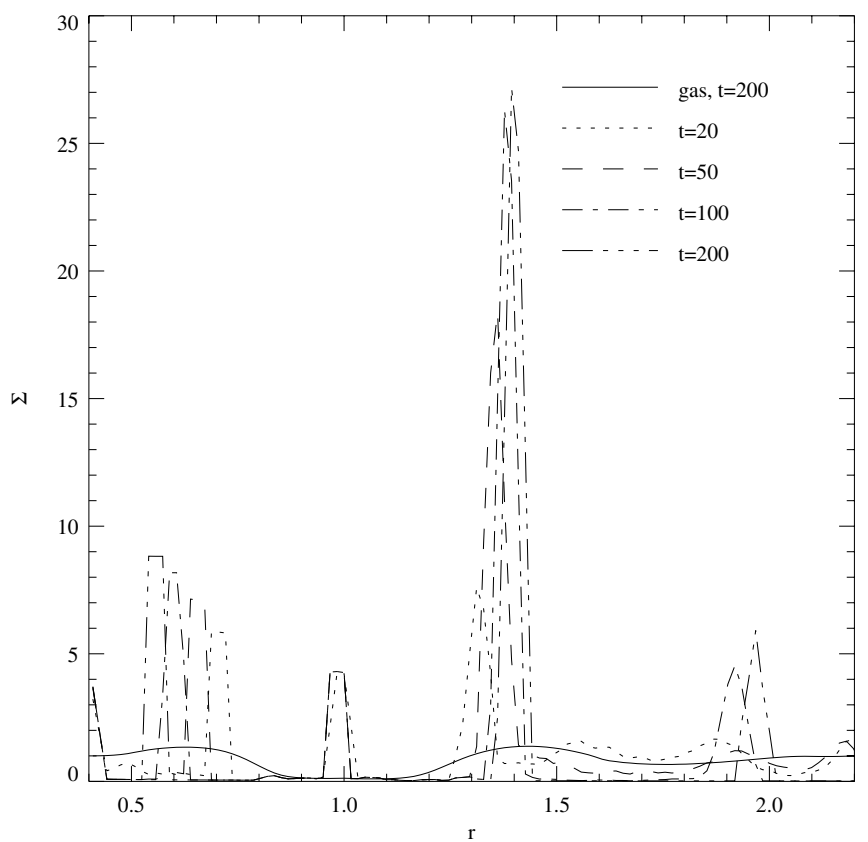

Fig. 14. Particle surface density $(s=37 \mathrm{~cm})$ around a $1 M_{\mathrm{J}}$ planet at different times.

In Fig. 14 we show the time evolution of the surface density of particles of $37 \mathrm{~cm}$. Four distinct features can be distinguished at $r \approx 0.6, r \approx 1, r \approx 1.4$ and $r \approx 1.9$, which are all due to 


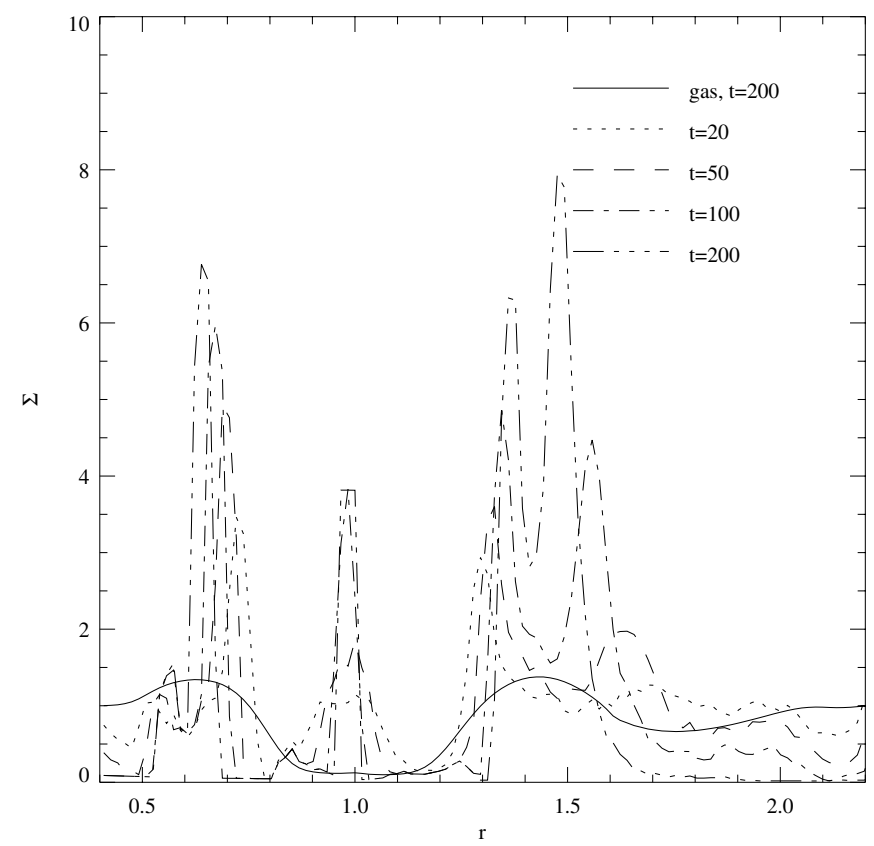

Fig. 15. Particle surface density $(s=1 \mathrm{~cm})$ around a $1 M_{\mathrm{J}}$ planet at different times.

the density structure in the disk. We have already mentioned the large peak near $r=1.4$, which builds up largely from the inside within 100 orbits. Note that the peak slowly moves outward but stops near $r=1.4$, because it has arrived at a pressure maximum.

For the peak near the inner edge of the gas gap at $r=0.6$ the situation is slightly different. Again, the peak is moving slowly but this time the density gradient inside $r=0.6$ is not strong enough to stop the particles completely. The peak slows down, but continues steadily towards the inner boundary and in the end it will move off the grid.

The peak at corotation is steady. Because the gas density has dropped approximately 2 orders of magnitude, the effective stopping time at $r=1$ has increased from 1.0 to 100.0. This means that it will take several hundreds of orbits more to remove this feature. Finally, a small outward moving feature can be seen associated with the density gradient near $r=1.9$. Note that resonant trapping is not important for particles of $s=37 \mathrm{~cm}$, which agrees with Fig. 7. However, the density gradients induced by the planet are so large that even particles of $1000 \mathrm{~cm}$ are not captured in the 2:1 MMR.

The behavior of particles of $1 \mathrm{~cm}$ is shown in Fig. 15. First thing to note is the absence of the density feature near $r=1.9$, which is due to the stronger gas drag that makes the particles follow the inward viscous motion of the gas. Near $r=1.4$ we can clearly see the two density peaks originating from both sides of the gas density maximum slowly moving towards each other. In contrast with the $37 \mathrm{~cm}$ particles from Fig. 14 the outer peak is the strongest, which is due to the lacking feature near $r=1.9$. Particles of $37 \mathrm{~cm}$ originally located at $r=1.8$ move outward, while their $1 \mathrm{~cm}$ counterparts move inward and contribute to the inward moving peak near $r=1.4$.

From Fig. 12 we conclude that the particles that had the largest accretion rate in Fig. 7 actually create the cleanest dust gaps. Already after 20 orbits the feeding zone is almost empty, and this has severe implications for the accretion rates. In fact, for all particles that open dust gaps the accretion rate equals zero after a finite amount of time.

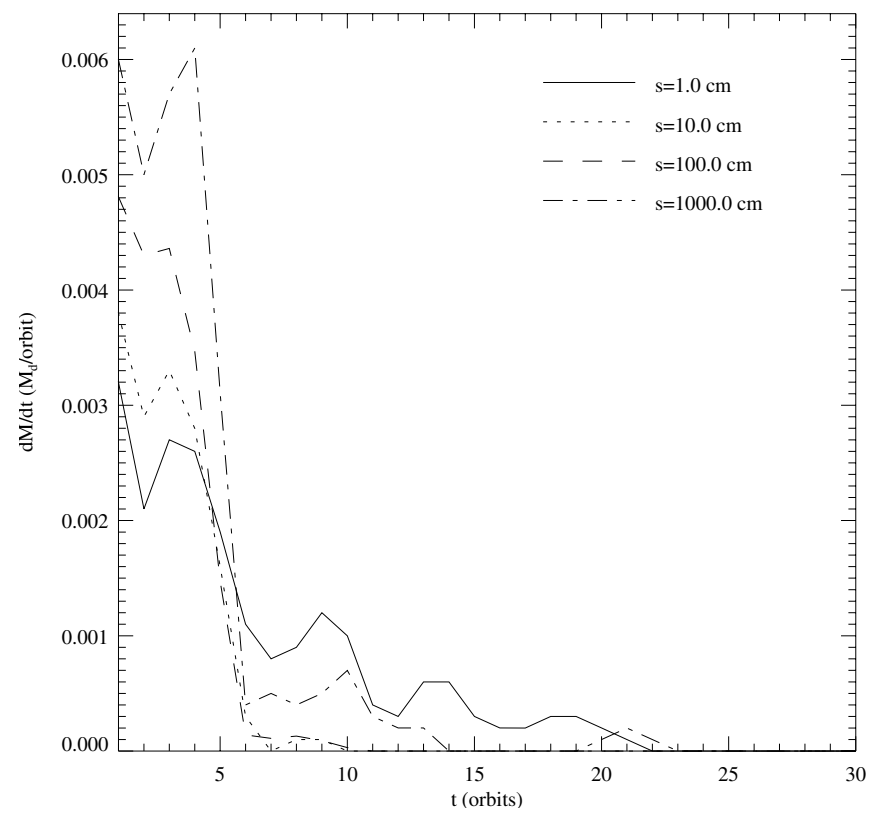

Fig. 16. Accretion of particles onto a $1 M_{\mathrm{J}}$ planet and an evolving gas disk for four different particle sizes. After 30 orbits, all accretion rates stay exactly zero.

This is illustrated in Fig. 16, where we show the accretion rate for four different particle sizes. After only 30 orbits, all accretion rates stay exactly zero. Note that this is unlike all previous models: for perfectly coupled particles as well as uncoupled particles there was still residual accretion after 200 orbits (see Fig. 5). This shows how powerful pressure structure in the disk is when it comes to moving dust particles.

The time scale at which accretion stops depends on the stopping time. The shortest time scales correspond to particles of 10 and $100 \mathrm{~cm}$, which have an initial stopping time of $T_{\mathrm{s}} \approx 1$. Smaller particles as well as larger particles have a smaller drift velocity (see Eq. (24)) and therefore it takes longer for them to clear the dust gap. Eventually, for the smallest particle sizes we expect to recover the gas accretion rate, while for the largest particle sizes we expect some residual accretion.

The accretion rate during the first 5 orbits of the planet grows monotonically with particle size. Particles of $1 \mathrm{~cm}$ accrete approximately as fast as perfectly coupled particles in this stage (see Fig. 3). Our resolution close to the planet is not high enough to see the Roche lobe clearing stage as was observed in Paardekooper \& Mellema (2006a) using an adaptive mesh. Larger particles accrete faster, and the particles of $1000 \mathrm{~cm}$ have an even higher accretion rate than uncoupled particles (see Fig. 6). The turnover occurs around $T_{\mathrm{s}}=1$, which marks the transition from gas drag dominated dynamics $\left(T_{\mathrm{S}}<1\right)$ and gravity dominated dynamics $\left(T_{\mathrm{s}}>1\right)$. During this stage, when the planet has not opened up a gas gap yet, gas drag is able to speed up accretion as long as we are in the gravity dominated regime. However, when the initial stopping time $T_{\mathrm{s}}<1$ gas drag really takes over and forces the particles to accrete at the same rate as the gas.

In Fig. 17 we show the accretion rate after 200 orbits of a $1 M_{\mathrm{J}}$ planet for various particle sizes. Again we see that the accretion rate is identically zero for a large range of sizes, while at both ends of the figure we approach the accretion rates of the limiting cases of perfectly coupled particles (left) and uncoupled particles (right). The dust accretion rates almost never become steady, unless they are identically zero or in the limiting case of 


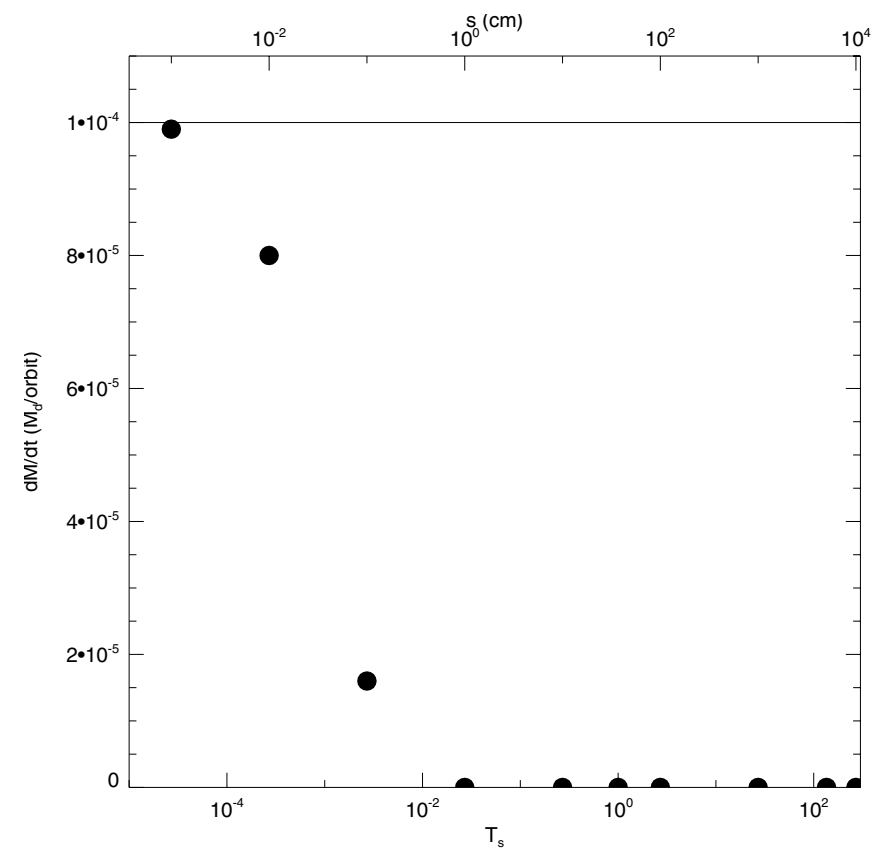

Fig. 17. Accretion of particles onto a $1 M_{\mathrm{J}}$ planet and an evolving gas disk after 200 orbits. The solid line gives the gas accretion rate. All runs included 5000 particles.

perfectly coupled particles (cf. Figs. 3 and 5). The radial distribution of the smallest particles evolves on a time scale proportional to $T_{\mathrm{s}}^{-1}$, which becomes prohibitively long when $T_{\mathrm{s}} \ll 1$. Therefore we chose a conservative approach to take the accretion rate after 200 orbits, when the gas accretion has settled to a steady state, as the final accretion rate for a given particle size, even though the particle accretion rate is still declining.

The transition from no accretion to gas accretion begins around $s=0.001 \mathrm{~cm}$, or equivalently $T_{\mathrm{s}}=0.000027$. This means that the total accretion flow onto the planet is severely depleted in particles larger than $10 \mu \mathrm{m}$. Even more, dust accretion never exceeds gas accretion, which makes it very difficult to enrich a giant planet in solids.

A ten times less massive planet of $0.1 M_{\mathrm{J}}$ does not open up a gas gap, and therefore the planet-induced pressure gradients in the disk are less strong. However, Paardekooper \& Mellema (2006a) showed that even for such a small planet dust accretion severely slows down. Figure 18 shows the dust accretion rates for various particle sizes. Again, we see accretion rates that are identically zero for $s>1.0 \mathrm{~cm}$, but the transition from gas accretion rates to zero accretion is shifted to larger $s$ with respect to the planet of $1 M_{\mathrm{J}}$. We find that particles larger than $100 \mu \mathrm{m}$ have significantly low dust accretion rates compared to the gas, which agrees with the result of Paardekooper \& Mellema (2006a). Also for this low-mass planet it is very hard to get enriched in solids.

More massive planets than Jupiter open up wider and deeper gaps, but the gap edges do not get much steeper. Therefore the minimum particle size that is able to move across the outer gap edge to be accreted by the planet is the same as for the $1 M_{\mathrm{J}}$ planet. The width gas gap as well as the dust gap increases, though, which brings the density peak at the outer gap edge close to the $2: 1 \mathrm{MMR}$. This could lead to interaction between large bodies trapped in the 2:1 MMR and smaller bodies trapped at the gap edge.



Fig. 18. Accretion of particles onto a $0.1 M_{\mathrm{J}}$ planet and an evolving gas disk after 200 orbits. The solid line gives the gas accretion rate. All runs included 5000 particles.

\section{Discussion}

When Weidenschilling \& Davis (1985) discovered that orbital resonances are effective dust traps, they argued that this would not stop dust accretion completely. The increase in eccentricity near the resonance would promote collisions between planetesimals, and the resulting debris would be small enough to move through the resonances towards the planet. Kary et al. (1993) showed that this is not the whole story: particles small enough to make it through the orbital resonances have a significant chance of being transferred to interior orbits rather than being accreted onto the planet. We have demonstrated that there is yet another barrier for smaller dust particles when the planet is massive enough to induce significant pressure gradients in the disk, which is already the case for a planet of $15 M_{\oplus}$ (Paardekooper \& Mellema 2006a).

According to Fig. 10, when a planetesimal breaks up into $100 \mathrm{~cm}$ sized boulders in a catastrophic collision, these can subsequently be accreted by a $1 M_{\mathrm{J}}$ planet. However, inspection of Fig. 17 reveals that $100 \mathrm{~cm}$ boulders are stopped at the outer gap edge, and they need to be grinded down to $10-100 \mu \mathrm{m}$ before they can reach the planet. Moreover, it becomes progressively harder to break smaller particles because they are better coupled to the gas and their relative velocities are low. This means that the accretion flow onto the planet will be severely depleted in solids. The same holds for a $0.1 M_{\mathrm{J}}$ planet, although somewhat larger particles may enter the accretion flow (see Fig. 18).

This has severe consequences for the final composition of the planet. According to Pollack et al. (1996), a $1 M_{\mathrm{J}}$ planet at 5.2 AU approximately spends the final 500000 years of its formation accreting the major part of its massive gaseous envelope. Before that happened, the planet had already reached a mass of $0.1 M_{\mathrm{J}}$, which makes it vulnerable to dust gap formation. Therefore the major part of the mass accreted by the planet is relatively dust-poor compared to the interstellar value, which is of importance for the final composition of the planet. When Jupiter starts accreting gas when its solid core as well as the gaseous 
envelope both have a mass of $10 M_{\oplus}$ the difference in solid content between dust-rich gas accretion and dust-poor gas accretion can be as large as $33 \%$. Therefore, the enrichment in solids in Jupiter requires traditional enrichment scenarios (e.g. core erosion Guillot et al. 2004; clathrate hydrate trapping Gautier et al. 2001a,b) should be much more effective than assumed until now.

Patterson (1987), in contrast with the suggestion by Weidenschilling \& Davis (1985), suggested that the accumulation of bodies in orbital resonances may lead to enhanced growth of planetesimals. Especially for a planet on an eccentric orbit, for which the eccentricities of the planetesimals in low-order resonances stay rather low. In either case, the size distribution of trapped particles may be very dynamical, because growth as well as destruction is enhanced with respect to the rest of the nebula. This makes it hard to predict the outcome of resonance trapping with respect to the resulting size distribution.

Another interesting question is what the change in opacity does to the accretion flow. Dust is the major source of continuum radiation in the disk, and if the dust content is very low the gas may not be able to cool efficiently through radiation. This may severely slow down gas accretion. The magnitude of the opacity drop depends on how many of the smallest dust particles enter the accretion flow, which in turn depends on the details of the collisions of the larger bodies.

We have focused on planets of relatively high mass, in order for the two- dimensional approach to be valid. For planets more massive than $0.1 M_{\mathrm{J}}$ the gas accretion rates in two and three dimensional simulations agree reasonably well (D'Angelo et al. 2003), and because the dust particles are probably confined to an even thinner layer we believe that three-dimensional effects will be of minor importance. Also the results presented here do not depend on the density structure within the Roche lobe of the planet.

Turbulence in circumstellar disks is probably of magnetic origin (Balbus \& Hawley 1990). If strong enough, it may prevent the formation of a deep gap but for reasonable disk parameters the gap formation paradigm is not changed (Papaloizou et al. 2004). Note that the only thing that is needed to trigger dust gap formation is the existence of planet-induced pressure gradients, and therefore as long as the turbulent disk structure does not overcome gap formation the mechanism presented here will operate to reduce dust accretion onto high-mass planets.

The effect of a nonzero eccentricity of the planet's orbit on resonance trapping was studied by Patterson (1987) and Kary \& Lissauer (1995). The effect on the gas disk, in particular the formation of a gap, is not different from case of a circular orbit (Papaloizou et al. 2001). Moreover, eccentricity growth of embedded planets is associated with deep annular gaps (Artymowicz 1992; Lin \& Papaloizou 1993; Goldreich \& Sari 2003; Sari \& Goldreich 2004; Kley \& Dirksen 2006). Therefore it is likely that dust accretion onto eccentric planets also suffers from the planet-induced pressure gradients in the disk.

In this paper, we have not considered migrating planets. Gapopening planets experience type II migration, for which the migration speed is comparable to the viscous inward drift of the gas. This migration rate is not enough to change the results described in the previous section. However, for planets that open up a shallow gas gap, the inward migration speed can be much higher. It is then possible that, depending on their size, particles will approach the planet both from the inner disk and the outer disk. The planet will overtake the smallest particles, while the particles that are less well-coupled to the gas still overtake the planet on their way in. This means that dust trapping in the inner resonances becomes possible for particles that are being overtaken by the planet. Furthermore, the inner gap edge acts as a dust barrier for particles coming from the inner disk in the same way as the outer gap edge does for particles coming from the outer disk. Therefore, also in the case of a migrating planet dust accretion can be slowed down significantly.

When a planet is slowly accreting gas and dust from the surrounding disk, it will very gradually approach the mass that is required to open up a gap, first only in the dust disk, later also in the gas disk. Before that time, only particles that are captured in resonances will not reach the planet. This applies to the largest particles. As the planet gains mass, it may capture smaller and smaller particles in resonances. Therefore, the maximum particle size that may accrete onto the planet is decreasing with planetary mass (see also Figs. 17 and 18).

For simplicity, we have chosen a constant initial surface density. When the density profile is steeper, the surface density in the outer disk will be lower than in the case considered here. Therefore, particles in the outer disk will be less well coupled, the effects described in the previous section will be even more pronounced.

\section{Summary and conclusion}

In this paper we have studied the accretion rates of solid particles of different sizes onto high-mass planets using a new particlebased method for the integration of the equations of motion of the dust component. We have shown that we can reproduce the gas accretion rates as found in hydrodynamical simulations in the limit of perfectly coupled particles. In the limit of no coupling to the gas, the planet is able to clear its feeding zone in approximately 100 orbits, regardless of the initial eccentricity distribution of the particles. In order for accretion to proceed it is necessary to continuously replenish the feeding zone.

For a non-evolving gas disk three different classes of particles can be distinguished: the smallest particles couple very well to the gas and they accrete at the same rate as the gas, the largest particles get trapped in exterior resonances with the planet, and only particles with intermediate sizes $\left(1 \mathrm{~cm} \leq s \leq s_{\text {min }}\right)$ accrete onto the planet at a higher rate than the gas.

When the gas is allowed to evolve dynamically it turns out that the planet-induced pressure gradients due to the formation of an annular surface density depression play a major role in the movement of dust particles towards the planet. Only the particles that couple very well to the gas are able to move across the outer edge of the density depression, which limits the size of particles that can be accreted by a $1 M_{\mathrm{J}}$ planet to $s \leq 10 \mu \mathrm{m}$. For planets of lower mass somewhat larger particles may be accreted, but even for a $0.1 M_{\mathrm{J}}$ planet $s \leq 100 \mu \mathrm{m}$. The lack of accreting large bodies may have important consequences for growing planets in disks, especially with regard to their enrichment in solids. If a large mass fraction of the solid component of the disk resides in particles larger than $10 \mu \mathrm{m}$ then it is not possible to enrich the planet.

\section{References}

Artymowicz, P. 1992, PASP, 104, 769

Balbus, S. A., \& Hawley, J. F. 1990, BAAS, 22, 1209

Blum, J., \& Muench, M. 1993, Icarus, 106, 151

Blum, J., \& Wurm, G. 2000, Icarus, 143, 138

Boss, A. P. 1997, Science, 276, 1836

Bryden, G., Chen, X., Lin, D. N. C., Nelson, R. P., \& Papaloizou, J. C. B. 1999, ApJ, 514, 344

Chambers, J. 2006, Icarus, 180, 496

D'Angelo, G., Henning, T., \& Kley, W. 2002, A\&A, 385, 647

D'Angelo, G., Kley, W., \& Henning, T. 2003, ApJ, 586, 540 
Dullemond, C. P., \& Dominik, C. 2005, A\&A, 434, 971

Duncan, M., Quinn, T., \& Tremaine, S. 1989, Icarus, 82, 402

Eulderink, F., \& Mellema, G. 1995, A\&AS, 110, 587

Garaud, P., Barrière-Fouchet, L., \& Lin, D. N. C. 2004, ApJ, 603, 292

Gautier, D., Hersant, F., Mousis, O., \& Lunine, J. I. 2001a, ApJ, 550, L227

Gautier, D., Hersant, F., Mousis, O., \& Lunine, J. I. 2001b, ApJ, 559, L183

Godon, P. 1996, MNRAS, 282, 1107

Goldreich, P., \& Sari, R. 2003, ApJ, 585, 1024

Goldreich, P., \& Ward, W. R. 1973, ApJ, 183, 1051

Greenberg, R., Hartmann, W. K., Chapman, C. R., \& Wacker, J. F. 1978, Icarus, 35,1

Greenzweig, Y., \& Lissauer, J. J. 1990, Icarus, 87, 40

Greenzweig, Y., \& Lissauer, J. J. 1992, Icarus, 100, 440

Guillot, T., Stevenson, D. J., Hubbard, W. B., \& Saumon, D. 2004, The interior of Jupiter, Jupiter, The Planet, Satellites and Magnetosphere, 35

Kary, D. M., \& Lissauer, J. J. 1995, Icarus, 117, 1

Kary, D. M., Lissauer, J. J., \& Greenzweig, Y. 1993, Icarus, 106, 288

Kley, W. 1999, MNRAS, 303, 696

Kley, W., \& Dirksen, G. 2006, A\&A, 447, 369

Kokubo, E., \& Ida, S. 1998, Icarus, 131, 171

Kokubo, E., \& Ida, S. 2000, Icarus, 143, 15

Kokubo, E., \& Ida, S. 2002, ApJ, 581, 666

Kwok, S. 1975, ApJ, 198, 583
Lin, D. N. C., \& Papaloizou, J. C. B. 1993, in Protostars and Planets III, 749 Lubow, S. H., \& D’Angelo, G. 2006, ApJ, 641, 526

Lubow, S. H., Seibert, M., \& Artymowicz, P. 1999, ApJ, 526, 1001

Mayer, L., Quinn, T., Wadsley, J., \& Stadel, J. 2004, ApJ, 609, 1045

Paardekooper, S.-J., \& Mellema, G. 2004, A\&A, 425, L9

Paardekooper, S.-J., \& Mellema, G. 2006a, A\&A, 453, 1129

Paardekooper, S.-J., \& Mellema, G. 2006b, A\&A, 450, 1203

Papaloizou, J. C. B., Nelson, R. P., \& Masset, F. 2001, A\&A, 366, 263

Papaloizou, J. C. B., Nelson, R. P., \& Snellgrove, M. D. 2004, MNRAS, 350, 829

Patterson, C. W. 1987, Icarus, 70, 319

Pickett, B. K., Cassen, P., Durisen, R. H., \& Link, R. 2000, ApJ, 529, 1034

Pickett, B. K., Mejía, A. C., Durisen, R. H., et al. 2003, ApJ, 590, 1060

Pollack, J. B., Hubickyj, O., Bodenheimer, P., et al. 1996, Icarus, 124, 62

Sari, R., \& Goldreich, P. 2004, ApJ, 606, L77

Schaaf, S. A. 1963, Handbuch der Physik (Berlin: Springer Verlag)

Shakura, N. I., \& Sunyaev, R. A. 1973, A\&A, 24, 337

Takeuchi, T., \& Lin, D. N. C. 2002, ApJ, 581, 1344

Weidenschilling, S. J. 1977, MNRAS, 180, 57

Weidenschilling, S. J., \& Davis, D. R. 1985, Icarus, 62, 16

Wisdom, J. 1980, AJ, 85, 1122

Woitke, P., \& Helling, C. 2003, A\&A, 399, 297

Youdin, A. N., \& Shu, F. H. 2002, ApJ, 580, 494 